\title{
Asymmetric Magneto-Impedance in CoFeSiB Amorphous Microwire
}

\author{
J. KRAVČÁK ${ }^{a, *}$ AND R. VARGA ${ }^{b}$ \\ ${ }^{a}$ Department of Physics, FEEI, Technical University of Košice, Košice, Slovakia \\ ${ }^{b}$ Institute of Physics, Faculty of Sciences, UPJŠ, Park Angelinum 9, 04154 Košice, Slovakia
}

\begin{abstract}
The symmetric dependences of magneto-impedance on the applied longitudinal dc magnetic field were measured in an amorphous CoFeSiB microwire. Additional application of a circumferential dc bias magnetic field was used to achieve the asymmetric magneto-impedance in the microwire. The obtained results are theoretically interpreted taking into account the cylindrical core-shell magnetic structure and the helical anisotropy induced during the microwire preparation. The observed very steep linear magneto-impedance dependences crossing $H=0$ are promising for technical applications of the CoFeSiB microwire as a sensor of low magnetic fields.

PACS numbers: 75.30.Gw, 75.50.Kj, 75.60.Ej
\end{abstract}

\section{Introduction}

In soft magnetic metals with transversal easy axis of magnetization when submitted to an external static magnetic field $H$ the magneto-impedance (MI) effect which consists in the change of the complex impedance $Z$ is observed. The first explanation of MI effect was in terms of classical skin effect in magnetic conductors with a large effective permeability and its strong dependence on the external dc magnetic field $H$ [1]. The quasistatic model based on the minimization of the free energy of a strip domain structure was presented [2], which does not take into account the dynamic effects related to the rapid magnetization motion. This model is successful in explaining the existence of peaks in MI field dependence and the MI frequency dependence in low frequency range.

\section{Theoretical background}

In the low frequency range, from $10 \mathrm{kHz}$ to $1 \mathrm{MHz}$, the driving alternating current $I_{\mathrm{ac}}$ flowing through the microwire generates a circular magnetic field $H_{\Phi}$. Such ac field causes a circular magnetic flux change and generates a longitudinal electric field that, in turn, gives rise to an induced voltage across the wire [3]. The basic idea of the quasistatic model is that in a metal microwire with circular magnetic permeability $\mu_{\Phi}$ the ac current $I_{\mathrm{ac}}$ is essentially confined to a surface layer with thickness given by the skin depth

$$
\delta=\sqrt{\frac{\rho}{\pi f \mu_{\Phi}}},
$$

where $\rho$ is the dc resistivity and $f$ - the frequency. A well known solution for the skin effect in a cylindrical conductor with radius $a$ [4] gives

$$
\frac{Z}{R_{\mathrm{dc}}}=k a \frac{J_{0}(k a)}{J_{1}(k a)} \approx S \frac{1+\mathrm{i}}{\delta},
$$

where $R_{\mathrm{dc}}$ is the dc resistance, $J_{0}$ and $J_{1}$ are the Bessel functions and $k=(1-\mathrm{i}) / \delta$ is the propagation constant. In case of a strong skin effect $(a \gg \delta)$ formula $(2)$ for

\footnotetext{
* corresponding author; e-mail: jozef.kravcak@tuke.sk
}

the unit length of the conductor can be simplified ( $S$ is the conductor cross-section area). The magnitude of the MI effect depends on the frequency $f$, a ratio of material properties $\mu_{\Phi} / \rho$ and also on the thickness of the sample.

\section{Results and discussion}

The MI dependence is usually defined as a ratio $\Delta Z / Z=\left[|Z(H)|-\left|Z\left(H_{\max }\right)\right|\right] /\left|Z\left(H_{\max }\right)\right|$, where $|Z|$ is the impedance modulus and $H_{\max }$ is the maximum of the static measuring field $H$, at which the sample is considered to be magnetically saturated. In our experiments $|Z|$ at the frequency $f=1 \mathrm{MHz}$ is given by $|Z|=U_{\mathrm{ac}} / I_{\mathrm{ac}}$, where $I_{\mathrm{ac}}$ is the amplitude of harmonic current flowing through the microwire and $U_{\mathrm{ac}}$ is the total voltage amplitude between two fixed points of the microwire. The saturation field was $H_{\max }=15 \mathrm{kA} / \mathrm{m}$.

An amorphous glass-coated ferromagnetic microwire with a metallic nuclei part diameter of $7 \mu \mathrm{m}$ (a total diameter of $9 \mu \mathrm{m}$ ) was prepared from alloy of the composition $\mathrm{Co}_{70.5} \mathrm{Fe}_{4.5} \mathrm{Si}_{15} \mathrm{~B}_{10}$ by quenching and drawing method [5]. The microwire exhibits vanishing but negative magnetostriction and the helical anisotropy induced during its production (quenching and drawing) [6]. The MI dependences, presented in Fig. 1, were measured at frequency of $1 \mathrm{MHz}$ with increasing ac current (circular magnetic field) amplitude $I_{\mathrm{ac}}$ from 0.1 to $10 \mathrm{~mA}\left(H_{\Phi}\right.$ from 4.4 to $440 \mathrm{~A} \mathrm{~m}^{-1}$ ). A gradual transition from double-peak to single-peak shape of MI dependences was recorded. The two symmetric peaks appear at axial field strengths $\pm H_{K}$, where a torque of effective anisotropy on magnetization is compensated by the dc field [4]. The increasing amplitude $I_{\text {ac }}$ shifts the peak positions $\pm H_{K}$ closer to zero. The hysteretic behaviour of MI was observed at low field strength $-H_{K}<H<H_{K}$. Formation of one symmetric peak takes place from $I_{\mathrm{ac}} \geq 6.7 \mathrm{~mA}$ (or $H_{\Phi} \geq 295 \mathrm{~A} \mathrm{~m}^{-1}$ ). The hysteresis, at low field strength, exists also on the single-peak MI dependence at $I_{\mathrm{ac}}=10 \mathrm{~mA}$ (or $H_{\Phi}=440 \mathrm{~A} \mathrm{~m}^{-1}$ ) and the jumps in MI peak (Fig. 1) are observed at $H= \pm 15 \mathrm{~A} / \mathrm{m}$. It is evident that ac current with amplitude $I_{\mathrm{ac}}=10 \mathrm{~mA}$ saturates the shell in the circular direction and in the presence of dc axial field, the jumps between two states 
with helical direction of the magnetization occur during the axial magnetization reversal [6].

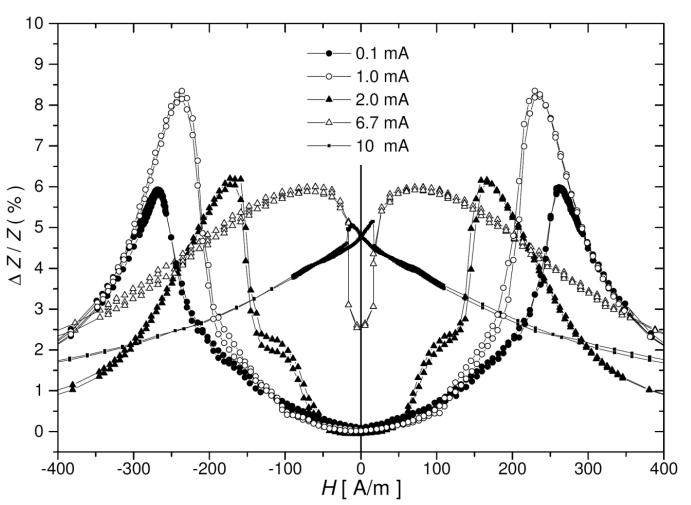

Fig. 1. Transition from double-peak to single-peak shape of MI dependences for different ac current (circular magnetic field) amplitude $I_{\mathrm{ac}}$.

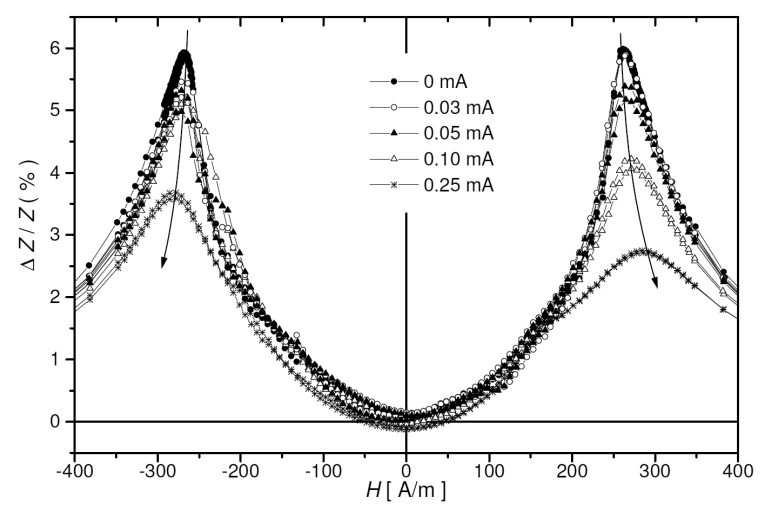

Fig. 2. The asymmetric MI effect during magnetization rotation measured at frequency of $1 \mathrm{MHz}$ with ac current (circular magnetic field) amplitude $I_{\text {ac }}$ of $0.1 \mathrm{~mA}$ $\left(H_{\Phi}=4.4 \mathrm{~A} \mathrm{~m}^{-1}\right)$ and applied dc bias current $I_{\mathrm{dc}}$ from 0 to $0.25 \mathrm{~mA}$.

Considering cylindrical core-shell magnetic domain structure in the microwire we assume that two dynamic magnetization processes affecting MI effect in CoFeSiB microwire take place at frequency of $1 \mathrm{MHz}$ :

1. when circular magnetic field amplitude is small $\left(I_{\mathrm{ac}}<1 \mathrm{~mA}\right.$ or $\left.H_{\Phi}<44 \mathrm{~A} \mathrm{~m}^{-1}\right)$, although the circular domain walls in a shell of the microwire can move reversibly within the potential wells, their displacement at $1 \mathrm{MHz}$ is very small and the rotation of magnetization vector in the shell dominates, which results in a double-peak behaviour of MI with zero contribution at $H=0$ and sharp maxima at $H= \pm H_{K}[2,4]$;

2. when circular magnetic field amplitude is high enough $\left(I_{\mathrm{ac}}>6.7 \mathrm{~mA}\right.$ or $\left.H_{\Phi}>295 \mathrm{~A} \mathrm{~m}^{-1}\right)$, the circular domain walls in the shell become free and an irreversible domain wall displacement occurs and the transition to a single peak behaviour of MI is observed.

When the additional dc bias current $I_{\mathrm{dc}}$ in the interval from 0 to $0.25 \mathrm{~mA}$ with $I_{\mathrm{ac}}=0.1 \mathrm{~mA}$ was applied, the asymmetric MI effect was observed (Fig. 2). The small $I_{\mathrm{dc}}=0.03 \mathrm{~mA}$ slightly diverts the magnetization vector from the easy direction given by helical anisotropy and this results in the hysteresis in the left MI peak (Fig. 2). The asymmetry becomes evident, when $I_{\mathrm{dc}} \geq I_{\mathrm{ac}}$, i.e., the microwire is strongly asymmetrically magnetized in one circular direction only. Consequently the hysteresis vanishes, the peak position $\pm H_{K}$ increases and the asymmetric MI magnitude decreases by about $40 \%$.

\section{Conclusions}

The relatively small magnitude of the MI effect of about $5-8 \%$ can be ascribed to the helical anisotropy existing in the as-quenched $\mathrm{CoFeSiB}$ microwire. This anisotropy manifests itself in hysteretic behaviour of MI dependences at $-H_{K}<H<H_{K}$ and in the jumps observed in MI dependence at $I_{\mathrm{ac}}=10 \mathrm{~mA}$ (or $H_{\Phi}=$ $440 \mathrm{~A} \mathrm{~m}^{-1}$ ). A very steep linear MI dependence crossing $H=0$ at amplitude $I_{\mathrm{ac}}=10 \mathrm{~mA}$ gives the possibility to scan magnetic field of low strength, e.g. earth magnetic field. Nevertheless a further thermal treatment of the microwire is required to transform the helical anisotropy to circular one in order to achieve the higher magnitude of MI [7]. Further manifestation of the helical anisotropy is the asymmetric MI effect observed in CoFeSiB microwire, especially when the microwire is strongly asymmetrically magnetized in one circular direction only, $I_{\mathrm{dc}} \geq I_{\mathrm{ac}}$.

\section{Acknowledgments}

This work was supported by the Slovak grant projects VEGA No. 1/0076/09, VEGA No. 1/0136/10 and NanoCEXmat No. ITMS 26220120019. We would like to thank prof. A. Zhukov for providing the samples.

\section{References}

[1] R.S. Beach, A.E. Berkowitz, Appl. Phys. Lett. 64, 3652 (1994).

[2] F.L.A. Machado, S.M. Rezende, J. Appl. Phys. 79, 6558 (1996).

[3] K. Mohri, IEEE Trans. Magn. 28, 3150 (1992).

[4] M. Knobel, M. Vázquez, L. Kraus, in: Handbook of Magnetic Materials: Giant Magneto-impedance, Ed. K.H.J. Buschow, Vol. 15, Elsevier Sci. B.V., Amsterdam 2003, p. 497.

[5] M. Vázquez, Physica B 299, 302 (2001).

[6] A. Chizhik, C. Garcia, A. Zhukov, P. Gawronski, K. Kulakowski, J. Gonzales, J.M. Blanco, J. Magn. Magn. Mater. 316, 332 (2007).

[7] J.D. Santos, R. Varga, B. Hernando, A. Zhukov, J. Magn. Magn. Mater. 321, 3875 (2009). 